\title{
Functional Status in a Teenager with Phenylketonuria from the ICF Perspective
}

\author{
Hasan Bingöl ${ }^{1} \quad$ Mintaze Kerem Günel${ }^{2}$ \\ 1 Vocational School of Health, Department of Therapy and \\ Rehabilitation, Mus Alparslan University, Mus, Turkey \\ 2 Division of Health Science, Department of Physiotherapy and \\ Rehabilitation, Hacettepe University, Ankara, Turkey \\ J Child Sci 2017;7:e14-e18.
}

\begin{abstract}
Address for correspondence Hasan Bingöl, Vocational School of Health, Department of Therapy and Rehabilitation, Mus Alparslan University, Mus, Turkey (e-mail: hesenbingol@gmail.com).
\end{abstract}

\begin{abstract}
Objective The case aims to present a teenager with phenylketonuria in terms of functional status, by using the World Health Organization's International Classification of Functioning, Disability, and Health (ICF) perspective.

Case Description A 17-year-old teenager with phenylketonuria, classified level $\mathrm{V}$ in accordance with the Functional Mobility Scale, was recruited in this study. To exhibit body structure, functional status, participation, activity, and environmental and individual factors, assessment tools classified according to ICF for Children and Youth major categories were implemented.

Result After the assessment tools were applied, we found various problems ranging

Keywords

- phenylketonuria

- ICF

- functional status

- activity

- participation from body structure, function, and activity, to participation. In addition to these problems, there were cognitive problems as well.

Conclusion Despite various physical problems, the teenager suffered from cognitive problems more, that is, compared with physical problems in the teenager with phenylketonuria, cognitive problems constituted a bigger barrier to participation in daily life.
\end{abstract}

\section{Introduction}

Phenylketonuria (PKU) is a metabolic disorder characterized by an increasing of levels of phenylalanine in the blood. Phenylalanine is an amino acid that is acquired through diet. ${ }^{1}$ Due to lack of phenylalanine hydroxylase enzyme that breaks down phenylalanine, this amino acid accumulate in the blood. $^{2}$ If PKU is not cured, phenylalanine might increase up to harmful levels in the blood, leading to intellectual disability and other serious health problems. Children with PKU must sustain a normal phenylalanine level to support normal growth and development and to avoid severe neurologic impairment. ${ }^{3}$ The level of serum phenylalanine must be often measured. Unless patients do not continue a special diet, hyperphenylalaninemia can result in eczema, hypopigmentation, cognitive impairment, anxiety, depression, and seizures. ${ }^{4}$ Historically, diet has been the compulsory approach of treatment, and mothers were recommended against breastfeeding infants. ${ }^{5}$ For this reason, after newborn screening results, it is necessary to contact with family. The child's protein requirements are met through a special formulation of synthetic amino acids. ${ }^{6}$ Current guidelines support maintaining this diet throughout the lifespan, even after most neuronal development is complete, to avoid potential deterioration in neurologic function. Pharmacologic therapy with sapropterin dihydrochloride, which was approved in the United States in the last decade, can also benefit. ${ }^{5}$ It reduces blood levels of phenylalanine by 10 to $80 \%{ }^{7}$ Less severe forms of this condition, sometimes called variant PKU and non-PKU hyperphenylalaninemia, have a smaller risk of brain damage. People with very mild cases may not require treatment with a low-phenylalanine diet. Patients with PKU received

May 17, 2017

accepted after revision

May 28, 2017
DOI https://doi.org/

10.1055/s-0037-1604024.

ISSN 2474-5871.
Copyright @ 2017 Georg Thieme

Verlag KG Stuttgart . New York
License terms

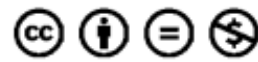


should be followed by a pediatric metabolic team composed of physicians, nurses, and dietitians. ${ }^{8}$ Mothers who wish to breastfeed infants with PKU should be encouraged to do so.

The occurrence of PKU varies among ethnic groups and geographic regions worldwide. In the United States, PKU occurs in 1 in 10,000 to 15,000 newborns. ${ }^{9}$ Most cases of PKU are detected shortly after birth by newborn screening, and treatment is started promptly.

İnsufficient physical growth has been defined in children with untreated PKU and in individuals treated in the past. ${ }^{10}$ Several last studies of the treatment of PKU suggest growth to be closer to normal, but the growth data as presented cannot be adequately compared with normal values. Until this time, convincing physical growth has not been satisfactory shown in children treated for PKU. Other symptoms including behavioral or social problems; seizures, shaking, or jerking movements in the arms and legs; stunted or slow growth; skin rashes such as eczema; small head size; a musty odor in urine that is a result of the extra phenylalanine in the body; and fair skin and blue eyes, due to the body's failure to transform phenylalanine into melanin, the pigment responsible for a person's color are present in individuals with PKU. ${ }^{11}$

As for physical characteristics or body composition of children with PKU, data are insufficient. However, several authors demonstrated that bone mineral density was lower in children with PKU than in typical developing individuals. $^{12}$ Previous studies have revealed that children with PKU have a decrease in bone mineralization compared with typical developing children. ${ }^{13}$

To our knowledge, no detailed studies are available related to muscle status and physical characteristics in patients with PKU. Thus, we can say that this case report is the first.

\section{Case Description}

E. Y. was a 17-year-old teenager with PKU. After birth he first received a diagnosis of lack of DHPRs (dehydrogenation receptors). DHPR is an L-type calcium channel of exterior membranes (surface membrane and T tubules), which acts as the voltage sensor of excitation-contraction coupling. ${ }^{14}$ Subsequently, he received his true diagnosis called phenylketonuria from other clinics. After reviewing his records, we decided to assess his uncommon condition. We communi- cated with the patient's family who applied to our hospital earlier on and then he was invited to our physiotherapy and rehabilitation unit for the evaluation of functional status.

Once the patient came in, we observed his posture and mobility level. E. Y. was ambulant, but had a bad posture problem. The most noticeable impairment was thoracic kyphosis and flexed neck. Also, we noticed that he had moderate cognitive problems, muscle weakness, and poor walking velocity, but he received commands easily. Before the actual start of the assessment, we received a verbal consent from the patient's mother.

\section{Assessment}

After we informed the patient's mother in detail, the mother approved to participate in our study and permitted us to evaluate her son. To evaluate a patient, we defined assessment tools then categorized these tools according to the World Health Organization's International Classification of Functioning, Disability, and Health for Children and Youth (ICF-CY) major categories (-Table 1). The ICF-CY checklist was used to verify assessment tools and outcomes.

\section{Description of Assessment Tools}

The Spinal Alignment and Range of Motion Measure (SAROMM) is a measure of posture and flexibility. Reliability and validity testing conducted for children with cerebral palsy (CP) is reported in developmental medicine and child neurology. This assessment form consists of three main subtests. The first one comprises evaluation of Cervical Spine, Thoracic Spine, Lumbar Spine, and Lateral Curve. ${ }^{15}$

In this examination, the child sits on a footstool with his/ her knees and hips at $90^{\circ}$ of flexion. At this position, the patient's upper body and back is observed carefully. The testing items were applied in order according to manual instructions.

The second subscale is regarding range of motion and muscle extensibility in the lower extremity. This subscale involves hip extension, hip flexion, hip abduction, hip adduction, hip external rotation, hip internal rotation, knee extension, hamstring extensibility, ankle dorsiflexion, and ankle plantar flexion.

Table 1 Assessment tools categorized by ICF-CY major categories

\begin{tabular}{|l|l|l|l|}
\hline \multirow{2}{*}{ Body function and structure } & Activity & \multirow{2}{*}{ Participation } \\
\cline { 2 - 4 } & Capacity & Performance & \\
\hline Muscle strength assessment & GMFM & & CFCS \\
\hline SAROMM & GMFCS & Gillette FAQ & PODCI \\
\hline & FMS & WeeFIM & \\
\hline & MACS & & \\
\hline
\end{tabular}

Abbreviations: CFCS, Communication Function Classification System; FAQ, Functional Assessment Questionnaire; FMS, Functional Mobility Scale; GMFM, Gross Motor Function Measure; GMFCS, Gross Motor Function Classification System; ICF-CY, International Classification of Functioning, Disability, and Health for Children and Youth; MACS, Manual Ability Classification System; SAROMM, Spinal Alignment and Range of Motion Measure; WeeFIM, Functional Independence Measure. 
The third subscale is about the upper extremity range of motion covering shoulder flexion, adduction, and internal rotation, elbow flexion, forearm pronation, and wrist and finger flexion.

For upper extremity testing, the patient sits with knees flexed on the edge of a bed and implemented test items.

The Gross Motor Function Measure (GMFM) is a standardized observational instrument designed and validated to measure the change over the time in gross motor function of children with $\mathrm{CP} .{ }^{16}$ It consists of 88 items concerning gross motor proficiency, ranging from supine to walking, running, and jumping. In this study, the items only belonging to walking, running, and jumping were implemented because the patient was ambulant and could perform easily the other subtests.

The Gillette Functional Assessment Questionnaire (FAQ) is a self- or proxy-report measure that involves a 10-level classification of ambulatory function (FAQ walking scale) and 22 functional locomotor activities rated on a 5-level Likert difficulty scale (FAQ 22-item skill set). The FAQ is used to assess a person with all levels of walking ability and focuses on what an individual can do independently with the use of assistive devices or orthotics as needed to maximize function. ${ }^{17}$ Assessment outcomes related to FAQ scale were obtained from caregiver based on proxy-report measure.

The Functional Independence Measure (WeeFIM) is an 18item, 7-level ordinal scale instrument that measures a child's consistent performance in essential daily functional skills. Three main domains (self-care, mobility, and cognition) are assessed by interviewing or observing a child's performance of a task to criterion standards. ${ }^{18}$ We obtained information regarding WeeFIM scale items from the mother.

The Functional Mobility Scale is an assessment tool characterizing functional mobility of children with CP. It is also an aid for communication between orthopaedic surgeons and health professionals, which is based on scores of functional mobility over three distinct distances, chosen to represent mobility in the home, at school, and in the wider community. ${ }^{19}$

The Communication Function Classification System (CFCS) is defined as everyday communication performance of an individual with $\mathrm{CP}$, which consists of five levels. The CFCS focuses on activity and participation levels as described in the World Health Organization's ICF. ${ }^{20}$

The Manual Ability Classification System (MACS) delineates how children with CP use their hands to handle objects in daily activities. MACS has five levels. The levels are based on the children's self-initiated abilities to handle objects and their need for assistance or adaptation to perform manual activities in daily life. The MACS brochure also describes differences between adjacent levels to make it easier to determine which level best corresponds with the child's ability to handle objects. ${ }^{21}$

\section{Pediatric Outcomes Questionnaire}

The Pediatric Outcome Questionnaire includes 86 items associated with participation, for example, eating, bathing, school work, and playing with friends. For each activity, the assessment score is different. Each of the functional evaluation can be graded within the ICF framework. Most of PODCI's measures comply within the Activities and Participation component under the International Classification of Functioning, Disability, and Health Performance framework. ${ }^{8,22}$

\section{Results}

After completing assessment, we documented the following outcomes respectively.

\section{With Respect to Body Function and Structure}

Spinal problems consisted of impairment in alignment and most of them could not be corrected passively. Posture was usually flexed due to existing rigid vertebral column, and there was limited mobility in vertebral column, especially in the upper back. Thus, the child was unable to correct his upper back.

Knee and hip joints showed mild limitation and hamstring in both side and were not flexible. Moreover, we did not find any restriction of ankle plantar flexion, ankle dorsiflexion, and upper extremities' range of motion.

Muscles strength was evaluated predominantly in the lower extremity and trunk muscles (Kendal scale). The child presented with mild-to-moderate muscle weakness. Weakness in trunk extensor was attributed to long-term thoracic kyphosis. However, this condition has not been affecting child's daily living very much. We could not comment on causes of other muscle weaknesses.

With Respect to Activity, Capacity, and Performance The total score for subtest of GMFM including walking, runnıng, jumpıng, and climbing stairs is 28 . Especially E. Y. presented with problems with advanced motor skills, such as walking forward 10 consecutive steps on a 2-cm straight line.

Gross Motor Function Classification System level was 1 because the patient was ambulant and was able to walk easily anywhere without any support.

In respect of functional mobility level, the teenager could walk independently on level surfaces and did not use walking aids or need help from another person (level 5).

Total points for WeeFIM scale is 102 , and the patient suffered more from comprehension, expression social interaction, problem solving, and memory. Assessment outcome for the FAQ corresponded to the S04 item, which refers to walking up and down the stairs without using the railing.

The patient's manual ability level was classified in accordance with the MACS scale and corresponded to level 1 , which means that the child merely had limitation in activity requiring speed and accuracy, but no limitations in daily living activities.

\section{With Respect to Participation}

In respect of everyday communication performance, the patient represented level 4 , classifying according to the CFCS. It means that the patient has some success as an effective sender and/or an effective receiver with familiar partners, whereas communication with unfamiliar people is not effective. 


\section{Pediatric Outcomes Data Collections Instruments}

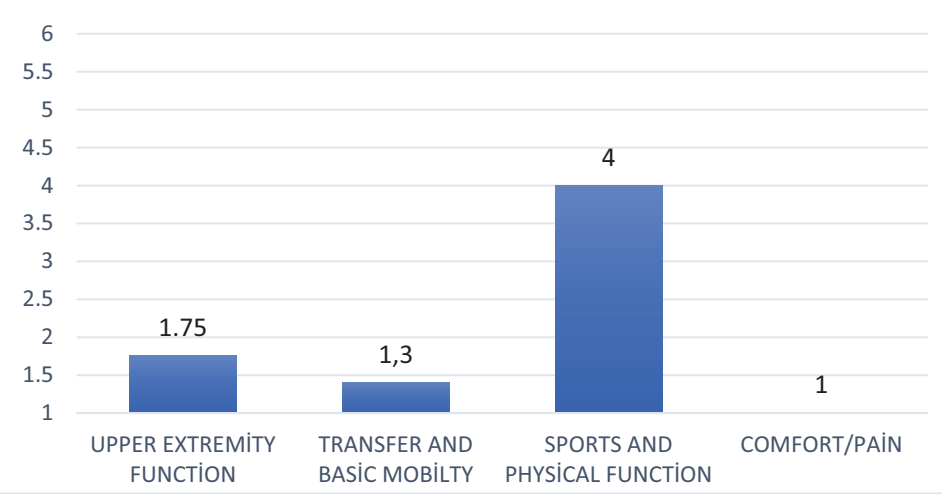

Fig. 1 Distribution of PODCl's items in accordance with participation. Items have different weights, with possible scores ranging from 3 (for response choices indicating "often," "sometimes," or "rarely or never") to 6 (for response choices indicating "none," "very mild," "mild,"

"moderate," "severe," or "very severe"). For most items, a lower number indicates higher functioning or a more positive quality of life. Each indication corresponds to a number from 1 to 6 or from 1 to 5.

\section{Pediatric Outcomes Data Collections Instruments}

As documented in - Fig. 1, the child had more problems with sports and physical function than with other categories. In contrast to this condition, he presented little problems in upper extremity function, transfer, and mobility. Comfort/ pain score indicated slight problem. The primary functional problem included advanced motor skills, but never limited his daily living extremely.

\section{Controlling of the Measurement Outcome}

The International Classification of Functioning, Disability, and Health Checklist Outcomes

As pointed out in - Fig. 2, the patient presented less problems in body function and structure than in activity and participation. This outcome was consistent with assessment outcomes of tools used in this study. Although there was no excessive impairment in body structure and function, the patient had considerable problems in activity and participation.

Environmental factors were evaluated as facilitator or barrier. Individual attitudes of immediate family members or housing services, systems and policies, social security, education and training services, income level of family, living in city, closeness to hospital, and so on are facilitator factors, whereas cognitive and emotional problems, societal attitudes, unfamiliar persons, opposite gender, and extended environment are barriers to participation.

\section{Conclusion}

The purpose of this study was to assess a child with PKU which occurs with an incidence of 1 in 2,600 living births in Turkey. ${ }^{23}$

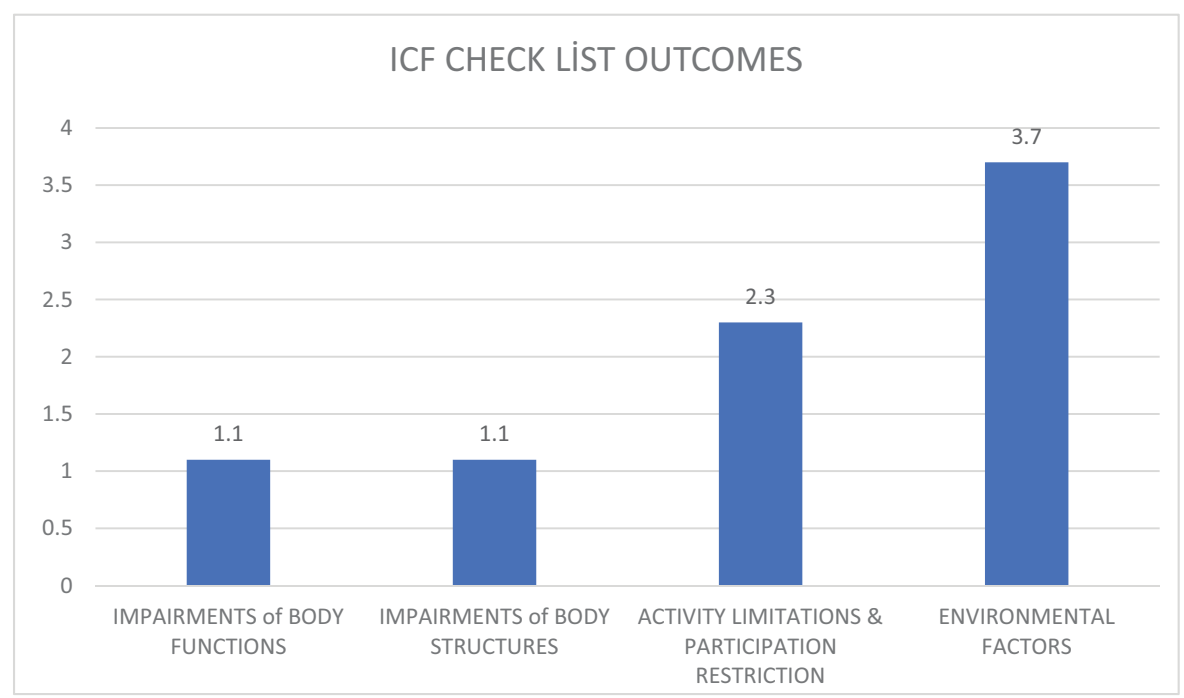

Fig. 2 International Classification of Functioning, Disability, and Health for Children and Youth outcomes data and mean values were calculated for each category. 
In conjunction with this purpose, the child with PKU was assessed, covering function and structures, activity, and participation. When the patient was evaluated, we considered not only physical status but also social aspects of the individual's functioning. PKU was not regarded only a metabolic disease. By taking contextual factors into account, the various assessment tools were applied on the teenager. With this perspective, external factors were assessed as well, for example, child's immediate family and their individual attitudes toward him. For this patient, the aforementioned factors were facilitators of participation in daily living and social integration. On the other hand, the individual's cognitive problems were barrier to participation in daily living and social integration.

Despite several physical problems that were identified, cognitive problems hindered participation more than physical problems.

Consequently, PKU should not be ignored by health professionals and especially by physiotherapist. The patient with PKU must be included in physiotherapy, rehabilitation, and cognitive educational program.

\section{Note}

At the time this article was written, Hasan Bingöl was a student at Institute of Health Science and Teaching Assistant at Mus Alparslan University.

\section{Conflict of Interest}

None.

\section{References}

1 Cederbaum S. Phenylketonuria: an update. Curr Opin Pediatr 2002;14(06):702-706

2 Vockley J, Andersson HC, Antshel KM, et al; American College of Medical Genetics and Genomics Therapeutics Committee. Phenylalanine hydroxylase deficiency: diagnosis and management guideline. Genet Med 2014;16(02):188-200

3 Keil S, Anjema K, van Spronsen FJ, et al. Long-term follow-up and outcome of phenylketonuria patients on sapropterin: a retrospective study. Pediatrics 2013;131(06):e1881-e1888

4 Harding CO, Winn SR, Gibson KM, Arning E, Bottiglieri T, Grompe M. Pharmacologic inhibition of L-tyrosine degradation ameliorates cerebral dopamine deficiency in murine phenylketonuria (PKU). J Inherit Metab Dis 2014;37(05):735-743

5 Sampson J, Groshong M, Parilo DM. What nurses need to know about PKU. Nursing 2016;46(08):66-67

6 Guest JF, Bai JJ, Taylor RR, Sladkevicius E, Lee PJ, Lachmann RH. Costs and outcomes over 36 years of patients with phenylketo- nuria who do and do not remain on a phenylalanine-restricted diet. J Intellect Disabil Res 2013;57(06):567-579

7 Herrmann ME, Brösicke HG, Keller M, Mönch E, Helge H. Dependence of the utilization of a phenylalanine-free amino acid mixture on different amounts of single dose ingested. A case report. Eur J Pediatr 1994;153(07):501-503

8 Acosta PB, Yannicelli S, Marriage B, et al. Nutrient intake and growth of infants with phenylketonuria undergoing therapy. J Pediatr Gastroenterol Nutr 1998;27(03):287-291

9 McBurnie MA, Kronmal RA, Schuett VE, Koch R, Azeng CG. Physical growth of children treated for phenylketonuria. Ann Hum Biol 1991;18(04):357-368

10 U.S. National Library of Medicine. Genetics Home Reference. Phenylketonuria. 2012. Available at http://ghr.nlm.nih.gov/condition/phenylketonuria

11 Holm VA, Kronmal RA, Williamson M, Roche AF. Physical growth in phenylketonuria: II. Growth of treated children in the PKU collaborative study from birth to 4 years of age. Pediatrics 1979; 63(05):700-707

12 Al-Qadreh A, Schulpis KH, Athanasopoulou H, Mengreli C, Skarpalezou A, Voskaki I. Bone mineral status in children with phenylketonuria under treatment. Acta Paediatr 1998;87(11): 1162-1166

13 Allen JR, Baur LA, Waters DL, et al. Body protein in prepubertal children with phenylketonuria. Eur J Clin Nutr 1996;50(03): 178-186

14 Felder E, Protasi F, Hirsch R, Franzini-Armstrong C, Allen PD. Morphology and molecular composition of sarcoplasmic reticulum surface junctions in the absence of DHPR and RyR in mouse skeletal muscle. Biophys J 2002;82(06):3144-3149

15 Mutlu A, Kelgökmen Z. Available at www.canchild.ca

16 CanChild Centre for Childhood Disability Research. Available at http://www.canchild.ca

17 Gorton GE III, Stout JL, Bagley AM, Bevans K, Novacheck TF, Tucker CA. Gillette Functional Assessment Questionnaire 22-item skill set: factor and Rasch analyses. Dev Med Child Neurol 2011; 53(03):250-255

18 Wong V, Wong S, Chan K, Wong W. Functional Independence Measure (WeeFIM) for Chinese children: Hong Kong Cohort. Pediatrics 2002;109(02):E36

19 Graham HK, Harvey A, Rodda J, Nattrass GR, Pirpiris M. The Functional Mobility Scale (FMS). J Pediatr Orthop 2004;24(05): 514-520

20 Hidecker MJC, Paneth N, Rosenbaum PL, et al. Developing and validating the Communication Function Classification System for individuals with cerebral palsy. Dev Med Child Neurol 2011; 53(08):704-710

21 Manual Ability Classification System for Children with Cerebral Palsy 4-18 years. Available at http://www.macs.nu

22 Barnes D, Linton JL, Sullivan E, et al. Pediatric Outcome Data Collection Instrument Scores in Ambulatory Children with Cerebral Palsy. J Pediatr Orthop 2008;28:97-102

23 Küçükkasap T. To Define Treatment, Diagnosis, Intervention and Screening in Phenylketonuria Disease in Turkey [master thesis]. 2013:7 